\title{
X-RAY MICROSCOPY AND SPECTROMICROSCOPY
}

\author{
G. MARGARITONDO \\ Institut de Physique Appliquée, Ecole Polytechnique Fédérale \\ PH-Ecublens, 1015 Lausanne, Switzerland
}

\begin{abstract}
Progress in the instrumentation and, in particular, in the photon sources makes it possible to implement a number of established X-ray spectroscopies in a high-lateral-resolution mode. We discuss the general trends of this field, and then we present a detailed analysis of a particular and very interesting branch: photoemission spectromicroscopy. The results include a recent world record in lateral and energy resolution, obtained by the MAXIMUM system at Wisconsin, and microimages of materials science systems as well as of neuron networks.
\end{abstract}

PACS numbers: $61.10 . \mathrm{Lx}, 79.60 .-\mathrm{i}$

\section{Introduction}

In the next three years, the world of X-ray research will be revolutionized by the advent of a handful of new sources of synchrotron radiation in the USA, in Europe and in Japan [1]. Perhaps the most relevant characteristic of these new sources will be their ultra-high brightness or brilliance [2]. This can be seen, for example, in the list of the table of source parameters for the storage ring ELETTRA in Trieste. The increase in brightness or brilliance will be of several orders of magnitude. Therefore, the complete exploitation of the new sources requires not a minor readjustment in the research programs, but a truly revolutionary rethinking of techniques and objectives.

It can be argued that microscopy, in a broad sense, is the area that will be most favored by the new levels of brightness or brilliance [1]. No matter what is the specific X-ray microscopic technique that one considers, all such techniques have a common need for high concentration of the photon beam. In turn, satisfying this need is made easier by the high source brightness.

The reason for this connection is quite fundamental [1]. The brightness [2] is a combination of the photon flux and of the photon beam geometrical characteristics. It increases as the flux increases, but also as the source area decreases and the beam's angular collimation increases. Neglecting losses, the brightness is conserved along a beamline (Liouville's theorem) $[1,2]$. 


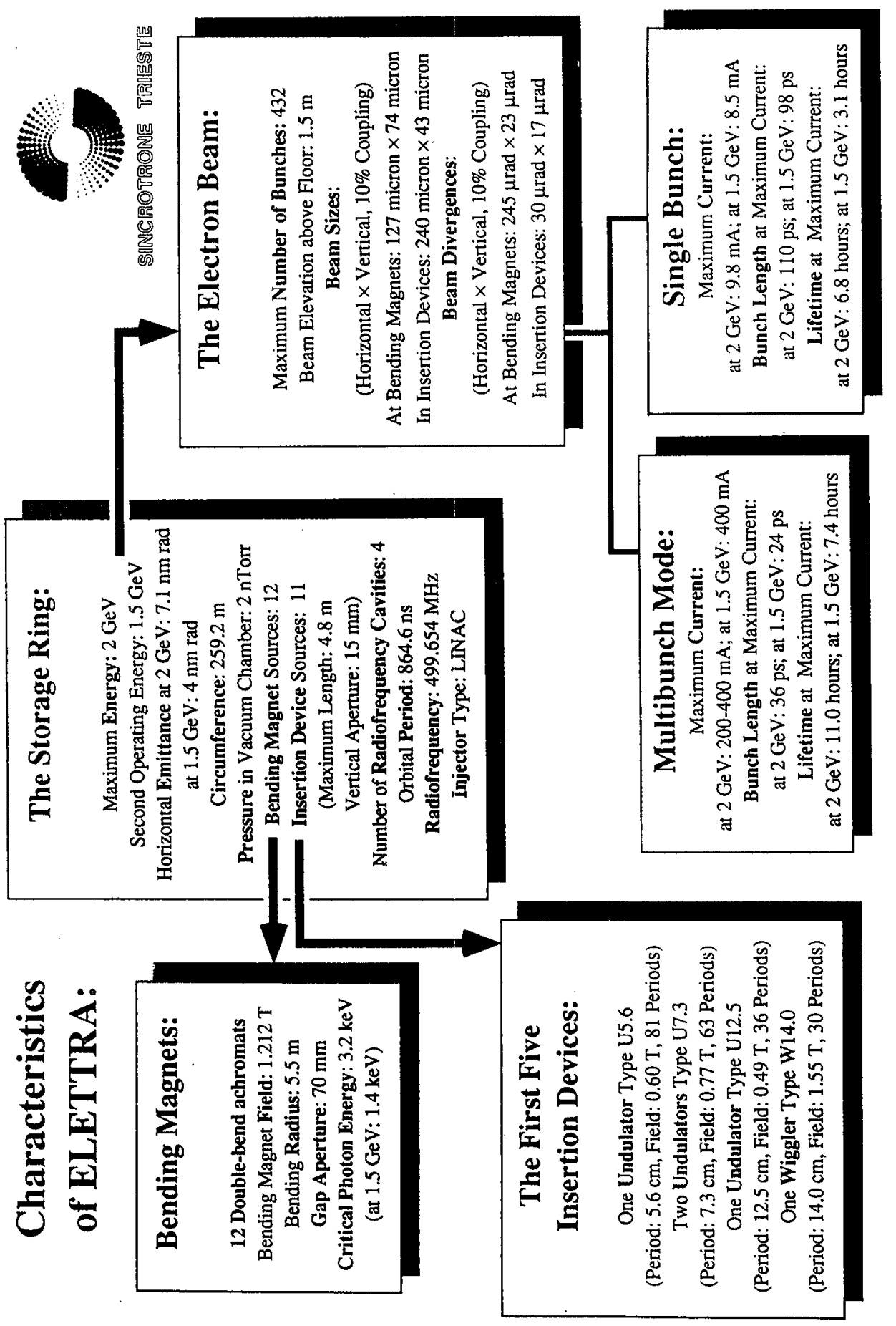


Let us consider, then, the task of focussing the beam, i.e. decreasing its size. This implies an increase in the angular divergence. If the source has a low brightness because of its large size and/or large angular divergence, such an increase may require the use of larger-size optical components, with an increase in cost and complexity. The best solution is to use a source with small size and high collimation, i.e. a source with high brightness.

Note that this need is common to all of the X-ray microscopy techniques. These fall into two categories, whose performances are quite complementary: optics imaging techniques and scanning techniques. In a scanning technique one focuses the photon beam and creates images by scanning it over the sample surface (typically, by moving the sample with respect to the beam); focussing is therefore an essential ingredient of the technique. But even in non-scanning microscopy, it is preferable to concentrate the photon flux into a small area, in order to increase the signal-to-noise ratio, hence again there is the need for focussing and high brightness.

The international scientific community makes a considerable effort to prepare itself for the advent of the new, ultra-high-brightness synchrotron sources, pushing to the limit the experiments with the present sources. One should mention two different kinds of experiments in this domain: microscopy and spectromicroscopy [1]. In the first case, processes stimulated by the $\mathrm{X}$-rays are used to create microscopic images. The ultimate resolution limit is linked as always to the wavelength of the primary particles (photons) and to the dimension of the optical components.

In the second case, the lateral-resolution capabilities of microscopy are coupled to the chemical and physical analysis capabilities of spectroscopy [1]. In essence, spectromicroscopy brings to the submicron domain a series of synchrotron radiation spectroscopies that have been used for years in the millimeter domain.

In this short overview, we will not be able to discuss in detail all of the different kinds of X-ray microscopies and spectroscopies that can take advantage of the new levels of brightness. In the next Section, we will present a partial list with brief descriptions. We will subsequently expand the discussion of one particular technique: photoemission spectromicroscopy - presenting some of the most recent data in that domain.

\section{Different kinds of X-ray microscopy and spectromicroscopy}

Virtually every kinds of interaction between X-rays and a solid system can be exploited in a microscopy/spectromicroscopy technique. Let us consider, for example, X-ray absorption. In its simplest form, it has been used for many years to produce microimages, for example, by detecting the $\mathrm{X}$-rays with a photoresist and then "reading" the images with an optical microscope [2]. But more sophisticated forms of absorption spectroscopy have recently found their way into microscopy.

For example, structural techniques such as EXAFS and XANES are now performed with high lateral resolution [3]. In particular, with the detection of secondary photoelectrons these techniques can be performed in a surface-sensitive version, so that one obtains at the same time high lateral resolution and high in-depth resolution [3]. 
Similarly, the scattering and diffraction of X-rays can be exploited for microscopy. Many of the corresponding techniques are not really new, but they will be greatly enhanced by the advent of ultra-bright sources $[2,4]$.

Quite new are the techniques based on particle emission with the notable exception of X-ray-stimulated fluorescence that has been used in a microscopy mode for a long time [2]. Other techniques of this kind are, for example, those based on X-ray-stimulated desorption of ions or neutral particles [2]. Because of the low signal level, however, photon-stimulated desorption spectromicroscopy of neutrals (based on the detection of photons emitted by the desorbed particules) is not feasible at the present time, whereas it will become feasible with ultra-high brightness.

\section{Scanning photoemission spectromicroscopy}

The photoelectric effect is, of course, one of the most important kinds of interaction processes between X-rays and materials [2]. Its importance is mainly related to the application of the corresponding photoelectron spectroscopy, which is at present the best probe of the electronic and chemical structure. The effectiveness of photoemission spectroscopy is due to the relative simplicity of the link between chemical properties and spectral features, and particularly between the chemical environment of an atom and the energies of the corresponding core-level features [2]. Such a link is much more direct than, for example, for multiple-electron phenomena like the Auger processes.

Unfortunately, in many systems of fundamental and/or industrial interest, the chemical and electronic properties change from place to place, and traditional photoemission is totally blind to changes that occur on a scale of less than a few tenths of a millimeter. Recently, electron microscopy has done amazing progress, all the way to the atomic-scale lateral resolution of the STM. But it is still affected by basic limitations; the STM, for example, has limited spectroscopic capabilities because of the limited energy domain that it can explore: chemical bonding affects a much wider energy range. Furthermore, they tend to be more interactive, that is perturbing, probes than photoemission.

Achieving good lateral resolution in photoemission spectroscopy is, therefore, a highly desirable objective. In the case of scanning photoelectron spectromicroscopy, the basic limit in lateral resolution is the same as for all of the X-ray focussing techniques. In essence, the resolution is determined by the aperture of the focussing device and by the wavelength. For practical cases, it is of the order of $10^{2}$ angstrom.

The major technical problem in reaching this resolution limit is the low signal level, so that in practical cases the lateral resolution is still set by the low signal. And the major technical problem in the implementation of scanning photoemission spectromicroscopy is focussing X-rays [1]. In a synchrotron-radiation photoemission experiment, one typically wants to enhance the surface sensitivity by decreasing the photoelectron escape depth, determined by its inelastic mean-free-path [2]. In turn, this is determined by the electrons' energy; the maximum surface sensi- 
tivity for electrons originating from the valence band requires photons of the order of a hundred electronvolt, i.e. soft X-rays that are quite hard to focus [1].

The difficulty is primarily related to the fact that these photons are neither transmitted nor reflected very effectively. Among many focussing devices that have been proposed to solve this problem, two have slowly emerged: the reversed Fresnel zone plate and the Schwarzschild objective (see Fig. 1) [1]. In the latter device, the normal-incidence reflectivity is enhanced by coating the spherical surface with a periodic multilayer, whose period determines the bandpass wavelength of the device.
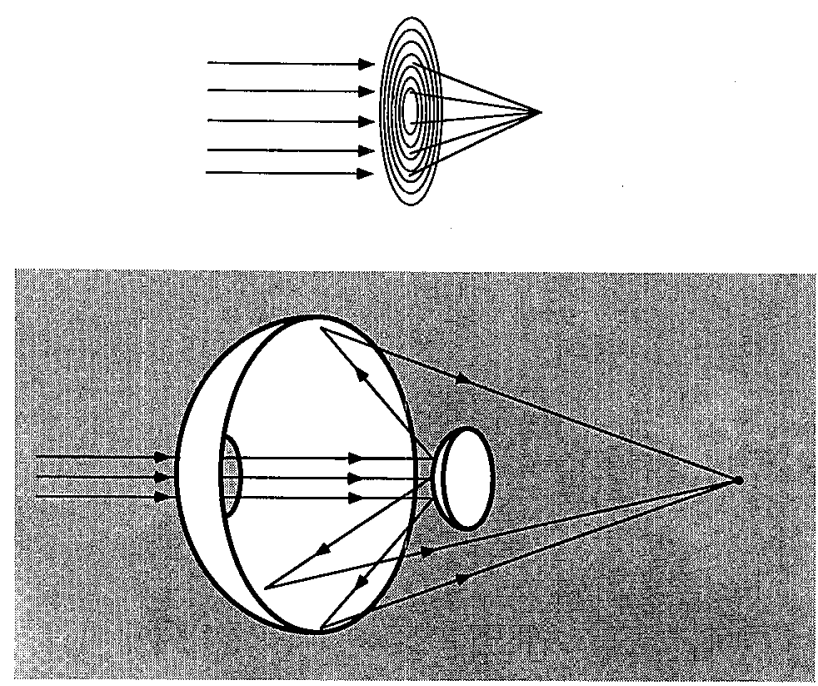

Fig. 1. Schematic representation of two focussing devices for soft X-rays, the reverse Fresnel zone plate (top) and the multilayer-coated Schwarzschild objective.

Recently, our Schwarzschild objective scanning photoemission spectromicroscope MAXIMUM $[5,6]$ has reached a record lateral resolution beyond 0.1 micron. This result is illustrated by Fig. 2. Here we see three-dimensional reconstructions of a photoelectron micrograph of a Fresnel zone plate. The plate has features of decreasing size, therefore by simple inspection it is possible to assess the minimum distance of distinguishable features in the micrograph.

The first image corresponds to the center of the zone plate, and it shows the quality of the image that one should expect from very easily distinguishable features. The second shows features near the resolution limit, i.e. beyond 0.1 micron. One can see that the quality of the imaged features is somewhat inferior than in the previous case, but still the features can be easily distinguished from each other.

These figures illustrate a very important point in photoemission spectromicroscopy: the image formation process includes topographic as well as chemical effects [5]. The topographic effects are related to the illumination of different sam- 

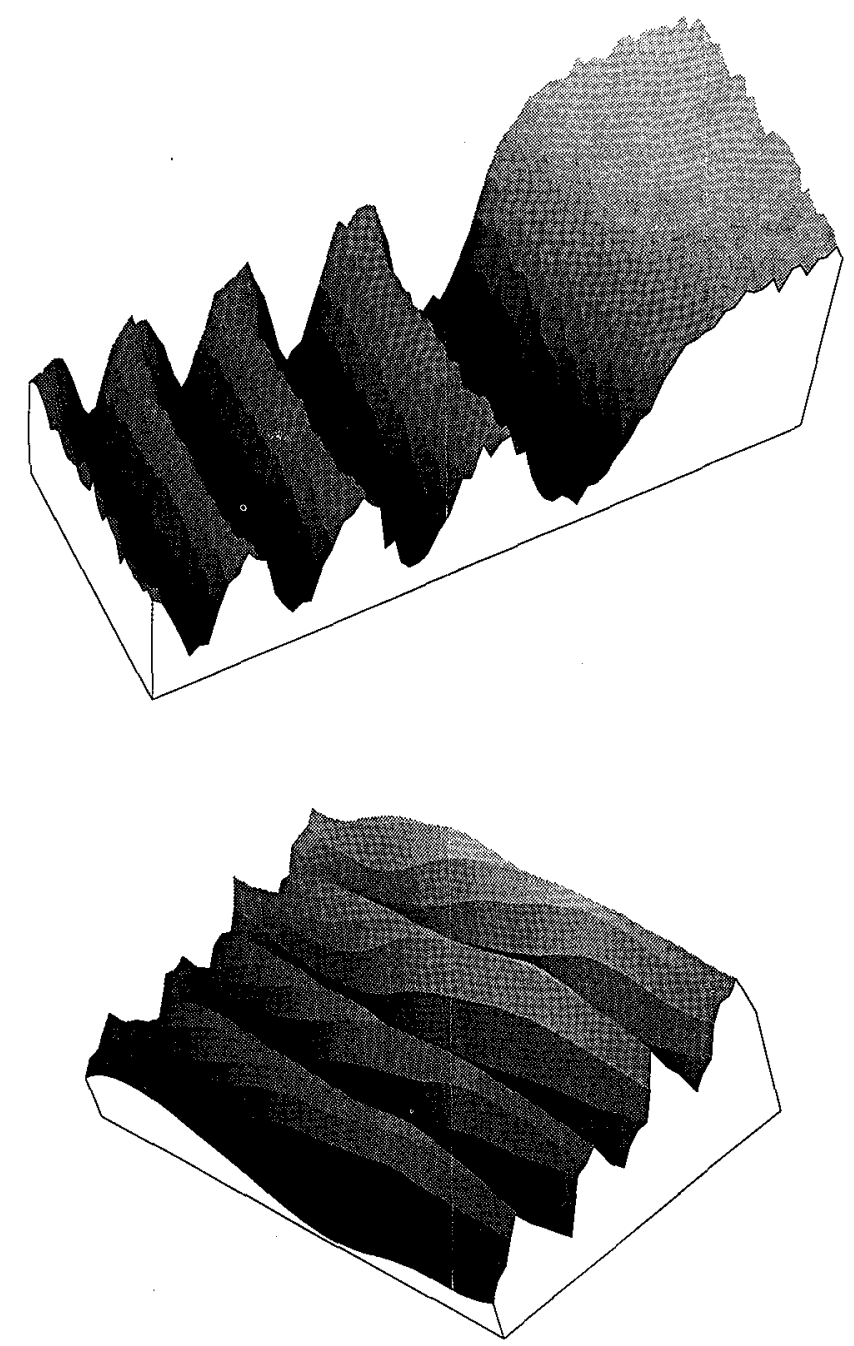

Fig. 2. Two three-dimensional reconstructions of photoelectron microimages taken with the MAXIMUM system. Both images correspond to portions of a Fresnel zone plate; the one on the top shows part of the central zone, whereas the other shows dome of the finest lines. The latter demonstrates a record lateral resolution beyond 0.1 micron. The three-dimensional reconstruction was performed with the Spyglass $\left.{ }^{(}\right)$software. Original data from F. Cerrina et al. [5].

ple areas by the X-ray beam as well as to the angular distribution of the photoelectrons. In general, the number of collected photoelectrons is higher for top flat surfaces than for lateral or curved surfaces.

The chemical aspect of the image formation process is evident in Fig. 3, which shows the reconstruction of the image of aluminum features on a silicon substrate. 
Note that these images were taken without energy-analyzing the photoelectrons. Recently, however, MAXIMUM has been operated in an energy-resolving mode, i.e. a true spectromicroscopy mode, with a record resolution of the order of hundreds of millielectronvolts.

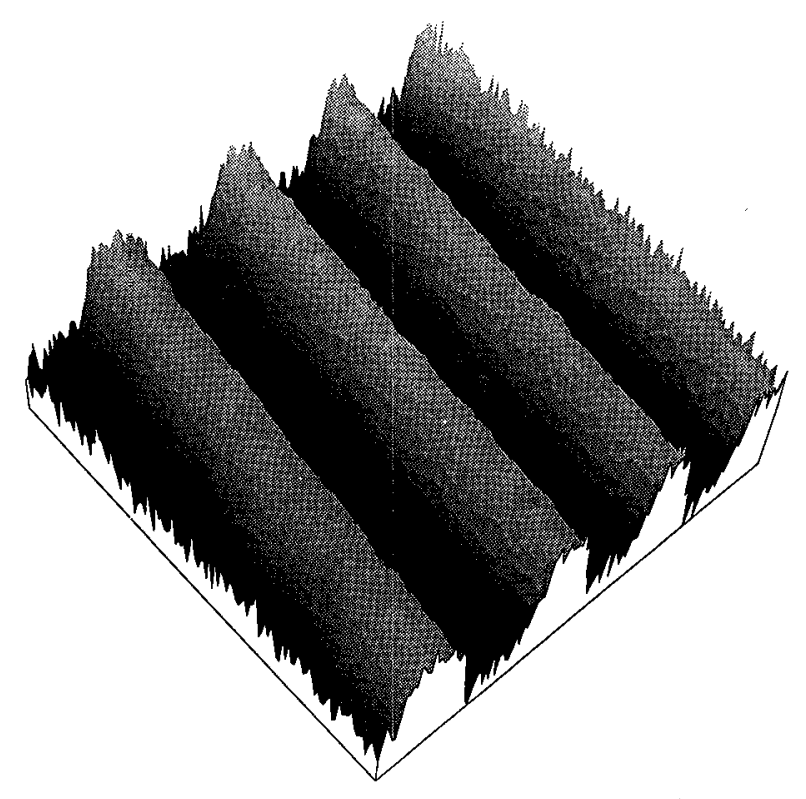

Fig. 3. Three-dimensional reconstruction of a series of $\mathrm{Al}$ lines on silicon, taken with 0.1-micron resolution with MAXIMUM. Original data from F. Cerrina et al. [5].

\section{Electron-optics photoemission spectromicroscopy}

The other mode of implementation of photoemission spectromicroscopy $[1,3]$ relies on an electron optical system to create microimages of portions of an area flooded with X-rays. One should note that this mode is not meant to replace scanning photoelectron spectromicroscopy, but rather to complement it $[1,3]$. The two approaches are in fact quite complementary in their capabilities, as it has been discussed in several previous reviews $[1,3]$.

And their complementarity extends to the present performances. For example, the electron-optics mode does not reach the lateral resolution levels that are possible at present with the scanning mode. On the other hand, it is capable of fast data-taking, which in turn makes it possible to take real-time video movies of the explored area, and to follow surface chemical reactions. On the contrary, the time per image is still quite long for scanning spectromicroscopy.

We would like to present some recent examples of results of the electron-optics mode in the specific area of biophysics [7]. There is a good reason for emphasizing biophysics: the micron-level resolution was a milestone in the path towards the 
application of photoemission techniques to the life sciences $[6,7]$. The reason is that the typical object that must be investigated is the cell, whose size is indeed of the order of microns. The present lateral resolution levels are also capable of imaging fine details of biological specimens such as the connecting parts of a neuron network.

Figure 4 shows a three-dimensional reconstruction of the photoelectron micrograph of a portion of a neuron network. The image was taken with the instrument X-ray secondary emission microscope (XSEM), designed and developed by Brian Tonner and his coworkers [3]. The specimen was prepared by Gelsomina De Stasio, Delio Mercanti and Maria Teresa Ciotti using an original method that produces high-quality samples suitable for studies under ultra-high vacuum [6-8].

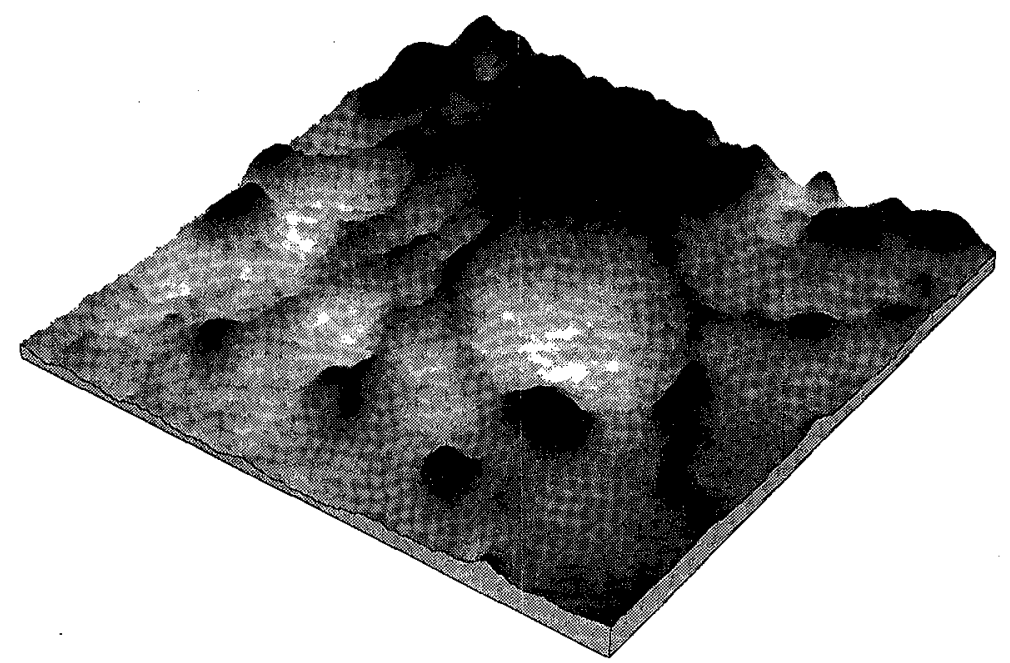

Fig. 4. Three-dimensional reconstruction of the photoelectron micrograph of a portion of a neuron network. The image was taken with the instrument $X$-ray secondary emission microscope (XSEM) at the Wisconsin Synchrotron Radiation Center. Original data from Ref. [7].

The XSEM gives microimages by means of an objective-projective electron optics system [3]. The electrons are revealed by a microchannel plate and by a videocamera system. The system can produce either real-time video images or high-resolution still frames. The data of Fig. 4 are part of a high-resolution image [7].

We can clearly see a central object, consisting of a glia cell, from which several connections depart, terminating in cones. The quality of the image suggests immediately the possibility to perform local microchemical analysis. And the first tests of this kind on neuron cells were indeed recently performed $[7,8]$.

The tests searched for evidence that the distribution of foreign metal atoms in a neuron network is not homogeneous. The spectroscopic technique in the case 
of the XSEM was XANES [3]. The results, primarily dealing with cobalt doping, clearly show inhomogeneities in the distribution. This demonstrates the need for a microchemical probe like photoemission spectromicroscopy to really understand many important effects of metal atoms in cells.

What are the advantages of this kind of chemical microanalysis with respect to alternate techniques? We have already seen the comparison with the STM. But microchemical analysis can also be performed, for example, with Auger techniques. There are nevertheless two big advantages in using photoemission-based techniques. First, the capability to detect small changes in the core-level chemical shifts, it means the capability to identify the chemical status of elements with high accuracy. Second, most Auger experiments use electrons as primary probes, and electrons are much more heavily perturbing than photons, leading to possible probe-induced damage of the observed specimen. A similar problem is present for most other electron spectroscopies.

\section{Future perspectives: what is new? Spectromicroscopy with and without synchrotron radiation}

One of the avenues of development of this novel field of spectromicroscopy is clearly set by the forthcoming commissioning of ultra-bright synchrotron sources. It is easy to predict that the experiments will take advantage from the increase brightness, first by reaching their natural lateral resolution limits. The increase in brightness, however, is much larger than what would be strictly necessary for this objective.

The additional increase can be exploited to improve other key performances, for example, to reach ultra-high energy resolution, or to obtain angular or spin resolution. It appears also feasible to push the time scale over which real-time experiment are possible, and go beyond the millisecond domain.

There is also a second avenue of development of the field of spectromicroscopy: the use of advanced laboratory sources. Rotating anodes have made substantial progress in recent years, reaching unprecedented power levels, which make possible to achieve a certain degree of lateral resolution coupled to a large spectral domain and to excellent energy resolution in photoemission.

Figures 5 and 6 shows a good example of these performances, obtained with the Scienta ESCA 300 spectromicroscope, recently commissioned at the Centre de Spectromicroscopie of the Ecole Polytechnique Fédérale de Lausanne [9]. In the first of these figures, we see evidence of a good resolving power (of the order of 5000) for the photoemission Fermi edge of silver taken with $\mathrm{Al} K_{\alpha}$ radiation. In the second, we see experiments performed with the lateral resolution (of the order of 25-30 micron) on a series of Au lines. The figure shows three-dimensional plots, in which the horizontal-plane axes correspond to space and energy, and the vertical one to photoemission intensity.

The plot clearly show the Au $f$-core levels, their intensity appearing and disappearing as the probe moves in and out of a gold stripe. Similar experiments have already revealed interesting inhomogeneities, for example, in fullerene films and in diamond-like films [9]. 


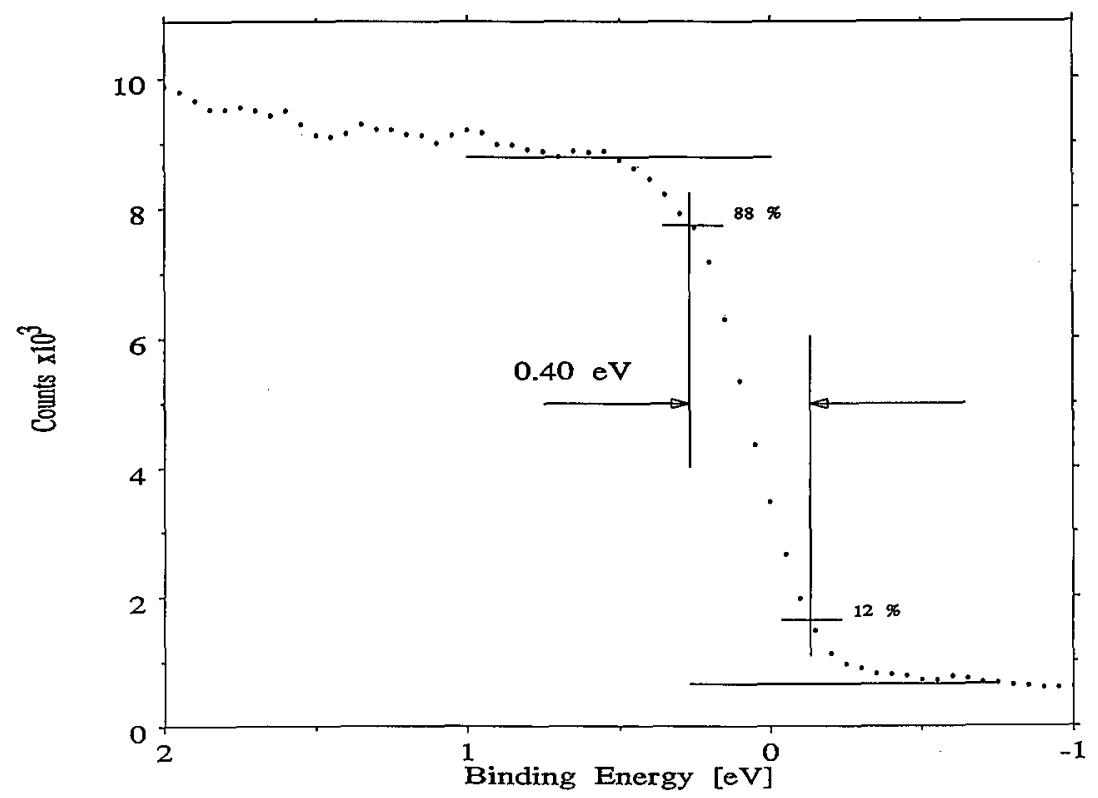

Fig. 5. High-energy-resolution spectrum of the Fermi edge of silver taken with $\mathrm{A} K_{\alpha}$ radiation using the Scienta ESCA 300 spectromicroscope of the Centre de Spectromicroscopie of the Ecole Polytechnique Fédérale de Lausannc. Original data from Ref. [9].

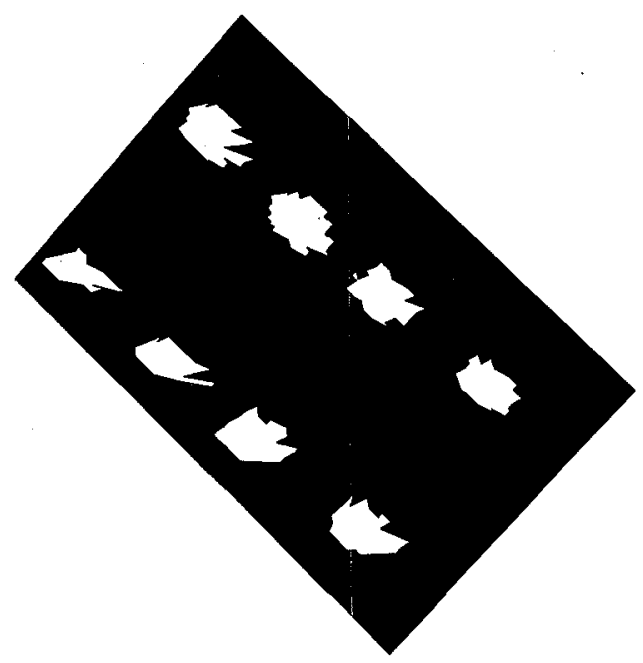

Fig. 6. Spectromicroscopy results obtained at the Centre de Spectromicroscopie of Lausanne, on a series of gold lines. The horizontal plane is an energy-space plane, whereas the vertical axis corresponds to the photoemission intensity. Therefore, we can scan horizontally over the energies, seeing the Au $f$-core peaks, or over the space along a line, seeing the appearance and disappearance of such peaks. Original data from Ref. [9]. 
In conclusion, this novel field of spectromicroscopy, and in particular photoemission spectromicroscopy, is rapidly expanding and replacing in part conventional photoemission. It should be emphasized that the present short review could cover only a portion of today's research in this field. Even considering photoemission spectromicroscopy alone, several other groups have made progress in the recent months. In Ref. [10] we present a partial list of recent articles in this field. All of these activities will be greatly enhanced by the advent of ultra-bright synchrotron sources in the near future. However, they do not need to be confined to specialized central laboratories, and therefore to a few selected experiments; laboratory-based spectromicroscopes offer a reasonable and largely complementary alternative, trading performance levels against flexibility [9].

\section{Acknowledgments}

Our activity in this field is funded by the Fonds National Suisse de la Recherche Scientifique, by the Ecole Polytechnique Fédérale de Lausanne, by the U.S. National Science Foundation both directly and through the operation of the Wisconsin Synchrotron Radiation Center, and by the Italian National Research Council. I am indebted to all of the colleagues with whom I collaborate in these activities: Franco Cerrina, Cristiano Capasso, Weiman $\mathrm{Ng}$, Ray Avjit-Chaudhury, Gelsomina De Stasio, Delio Mercanti, Maria Teresa Ciotti, Ruper Perera, Jim Underwood, Jeff Kortright, Brian Tonner, Scott Koranda, Carlo Coluzza, Fabia Gozzo, Philippe Almćras, and many others. It is always a pleasure to work with them in building this new and exciting field.

\section{References}

[1] G. Margaritondo, F. Cerrina, Nucl. Instrum. Methods Phys. Res. A 291, 26 (1990).

[2] G. Margaritondo, Introduction to Synchrotron Radiation, Oxford, New York 1988; G. Margaritondo, in: Annuario 1988/89 della Enciclopedia della Scienza e della Tecnica (EST), Mondadori, Milano 1988, p. 113.

[3] B.P. Tonner, Nucl. Instrum. Methods Phys. Res. A 291, 60 (1990); B.P. Tonner, G.R. Harp, Rev. Sci. Instrum. 59, 853 (1988); G.R. Harp, B.P. Tonner, in: Synchrotron Radiation in Materials Research, MRS Proceedings, Vol. 143, 279 (1989); B.P. Tonner, G.R. Harp, J. Vac. Sci. Technol. A 7, 1 (1989); G.R. Harp; Z.L. Han, B.P. Tonner, J. Vac. Sci. Technol. A 8, 2566 (1990); G.R. Harp, Zhi-Lan Han, B.P. Tonner, Phys. Scr. Vol. T 31, 25 (1990).

[4] V. Valkovic, private commun.

[5] F. Cerrina, B. Lai, C. Gong, A. Ray-Chaudhuri, G. Margaritondo, M.A. Green, H. Höchst, R. Cole, D. Crossley, S. Collier, J. Underwood, L.J. Brillson, A. Franciosi, Rev. Sci. Instrum. 60, 2249 (1989); F. Cerrina, S. Crossley, D. Crossley, C. Gong, J. Guo, R. Hansen, W. Ng, A. Ray-Chaudhuri, G. Margaritondo, J.H. Underwood, R. Perera, J. Kortright, J. Vac. Sci. Technol. A 8, 2563 (1990); W. Ng, A.K. Ray-Chadhuri, R.K. Cole, S. Crossley, D. Crossley, C. Gong, M. Green, J. Guo, R.W.C. Hansen, F. Cerrina, G. Margaritondo, J.H. Underwood, J. Korthright, R.C.C. Perera, Phys. Scr. 41, 758 (1990); C. Capasso, A.K. Ray-Chaudhuri, W. Ng, S. Liang, R.K. Cole, J. Wallace, F. Cerrina, G. Margaritondo, J.H. Underwood, J.B. Kortright, R.C.C. Perera, J. Vac. Sci. Technol. A 9, 1248 (1991). 
[6] G. De Stasio, W. Ng, A.K. Ray-Chaudhuri, R.K. Cole, Z.Y. Guo, J. Wallace, G. Margaritondo, F. Cerrina, J. Underwood, R. Perera, J. Kortright, D. Mercanti, M.T. Ciotti, Nucl. Instrum. Methods Phys. Res. A 294, 351 (1990); D. Mercanti, G. De Stasio, M.T. Ciotti, C. Capasso, W. Ng, A.K. Ray-Chaudhuri, S.H. Liang, R.K. Cole, Z.Y. Guo, J. Wallace, G. Margaritondo, F. Cerrina, J. Underwood, R. Perera, J. Kortright, J. Vac. Sci. Technol. A 9, 1320 (1991); G. De Stasio, C. Capasso, W. Ng, A.K. Ray-Chaudhuri, S.H. Liang, R.K. Cole, Z.Y. Guo, J. Wallace, F. Cerrina, G. Margaritondo, J. Underwood, R. Perera, J. Kortright, D. Mercanti, M.T. Ciotti, A. Stecchi, Europhys. Lett. 16, 411 (1991).

[7] G. De Stasio, P. Perfetti, S.F. Koranda, B. Tonner, J. Harp, D. Mercanti, M.T. Ciotti, G. Margaritondo, unpublished.

[8] G. De Stasio, P. Perfetti, N. Oddo, P. Galli, D. Mercanti, M.T. Ciotti, S.F. Koranda, S. Hardcastle, B.P. Tonner, G. Margaritondo, unpublished.

[9] C. Coluzza, F. Gozzo, P. Alméras, R. Sanjines, H. Jotterand, G. Margaritondo, unpublished.

[10] H. Ade, J. Kirz, S. Hulbert, E. Johnson, E. Anderson, D. Kern, Nucl. Instrum. Methods Phys. Res. A 291, 126 (1990); J. Kirz, H. Rarback, Rev. Sci. Instrum. 56, 1 (1985); H. Rarback, D. Shu, S.C. Feng, H. Ade, J. Kirz, I. McNulty, D.P. Kern, T.H.P. Chang, Y. Vladimirsky, N. Iskander, D. Attwood, K. McQuaid, S. Rothman, Rev. Sci. Instrum. 59, 52 (1988); D. Attwood, Y. Vladimirsky, D. Kern, W. Meyer-Ilse, J. Kirz, S. Rothman, H. Rarback, N. Iskander, K. McQuaid, H. Ade, T.H.P. Chang, OSA Proc. on Short Wavelength Coherent Radiation: Generations and Applications, Optical Society of America, Washington 1988, p. 274; Y. Vladimirsky, D. Kern, W. Meyer-Ilse, D. Attwood, Appl. Phys. Lett. 54, 286 (1989); H. Ade, J. Kirz, H. Rarback, S. Hulbert, E. Johnson, D. Kern, P. Chang, Y. Vladimirsky, in: X-Ray Microscopy II, Eds. D. Sayre, M. Howells, J. Kirz, H. Rarback, Springer, New York 1987, p. 280; P.L. King, A. Borg, C. Kim, P. Pianetta, I. Lindau, G.S. Knapp, M. Keenlyside, R. Browning, Nucl. Instrum. Methods Phys. Res. A 291, 19 (1990); C. Kunz, A. Moewes, G. Roy, H. Sievers, J. Voss, H. Wongel, HASYLAB Annual Report 1987, HASYLAB, Hamburg 1988, p. 366; R. Nyholm, M. Erikkson, K. Hansen, O.-P. Sairanen, S. Werin, A. Flodström, C. Törnevik, T. Meinander, M. Sarakontu, private commun.; D.W. Turner, I.R. Plummer, H.Q. Porter, Philos. Trans. R. Soc. Lond. A 318, 219 (1986); F. Pollack, S. Lowenthal, in: $X$-Ray Microscopy, Eds. G. Schmal, D. Rudolph, Springer, Berlin 1984, p. 251; J. Kirschner, ibid., p. 304; G. Beamson, H.Q. Porter, D.W. Turner, Nature 290, 556 (1981); M.S. Foster, J.C. Campuzano, R.F. Willis, J.C. Dupuy, J. Microsc. 140, 395 (1985); H. Petersen, W. Braun, E.-E. Koch, D. Rudolph, SPIE Proc. 733, 428 (1987); L. Beaulaigue, G. Jennings, R. Kampwirth, J. Kang, J.C. Campuzano, private commun. 